\title{
Entanglement of two distant Bose-Einstein condensates by detection of Bragg-scattered photons
}

\author{
B. Deb \\ Department of Materials Science, and Raman Center for Atomic, Molecular and Optical Sciences, \\ Indian Association for the Cultivation of Science, Jadavpur, Kolkata 700032, India \\ G. S. Agarwal \\ Department of Physics, Oklahoma State University, Stillwater, Oklahoma 74078, USA \\ (Received 11 March 2008; revised manuscript received 20 June 2008; published 31 July 2008)
}

\begin{abstract}
We show that it is possible to generate entanglement between two distant Bose-Einstein condensates by detection of Hanbury-Brown-Twiss-type correlations in photons Bragg scattered by the condensates. Upon coincident detection of two photons by two detectors, the projected joint state of two condensates is shown to be non-Gaussian. We verify the existence of entanglement by showing that the partially transposed state is negative. Further, we use the inequality in terms of higher-order moments to confirm entanglement. Our proposed scheme can be generalized for multiple condensates and also for spinor condensates with Bragg scattering of polarized light, with the latter capable of producing hyperentanglement.
\end{abstract}

DOI: 10.1103/PhysRevA.78.013639

PACS number(s): 03.75.Gg, 03.65.Ud, 32.80.-t, 42.50.Dv

\section{INTRODUCTION}

Quantum entanglement means inseparability of the joint wave functions of two or more distant objects into a product of wave functions of individual objects-even in the absence of any mutual interaction or communication between them. This epitomizes the underlying nonlocal character of the quantum world. One of the consequences of this nonlocal realism is that a single local measurement cannot reveal the complete state of an entangled system, since the process of measurement itself forces the wave function to "collapse" into one its measured (eigenstate) state in a probabilistic sense. Thus, the measurement process can post-selectively play a role in creation and manipulation of entanglement, and this is the essence of what is called "projective measurement."

Efficient generation of entanglement in many-particle systems and its robust transmission and transfer to other systems is important for quantum-information processes. Based on atom-photon interactions and the exchange of photons between the qubits, entanglement in distant atomic states [1-3] and also between photons $[4,5]$ has been experimentally demonstrated. There is another way of entangling two remote systems without requiring any direct interaction between them: This is based on projective measurement. This indirect method of creating entanglement between distant systems can also be applied for many quantum communication tasks. In a recent experiment, Moehring et al. [6] have created entanglement between two distant trapped ions by coincident detection of two photons spontaneously emitted by the two ions. An earlier experiment has shown interference of light emitted by two atoms [7-9], making use of projective measurements. Thiel et al. [10] have proposed a scheme of entangling several remote atomic qubits and thereby creating a Dicke state [11] of many atoms by projective measurement of photons using multiple photodetectors. Dicke states are particularly important for their robustness against particle loss $[12,13]$ and nonlocal properties of entangled multipartite states $[5,14-16]$. There are several other proposals for projecting distant noninteracting particles into entangled states via photodetection [17-22]. Continuous variables like the quadratures of a field mode (which are analogous to position and momentum) have also been employed [23] in entanglement studies.

The Bose-Einstein condensate (BEC) is a macroscopic quantum object where entanglement arises quite naturally due to two-body interactions. Bogoliubov theory [24] of Bose condensation reveals that in the ground state of condensates, two particles with opposite momentum are maximally entangled [25] in momentum variables as in an Einstein-Podolsky-Rosen (EPR) state [26]. This unique feature makes Bose condensates a good source of entanglement in motional degrees of freedom. Furthermore, in a twocomponent BEC, one can generate entanglement in hyperfine spin degrees of freedom [27-33]. In order to extract the intrinsic entanglement of a BEC for the useful purpose of quantum-information processes, it is required to excite quasiparticles in momentum modes by stimulated Raman scattering or Bragg scattering $[34,35]$. Then a scattered atom becomes entangled with Bragg-scattered photon [36]. In fact, Bragg spectroscopy can be used as a tool for generating entanglement of different kinds in a variety of physical situations. For instance, tripartite entanglement among two momentum modes of BEC and one electromagnetic field mode can be produced [25]. Furthermore, it has been shown that when a common laser beam passes through two spatially separated condensates, the photon scattered by the first condensate carries and transfers quantum information to the second one and thereby two condensates become entangled [37]. A similar experimental scheme has been used to produce and subsequently measure the phase difference between two spatially separated condensates [38].

Here we propose a scheme for generation of entanglement between two remote condensates by projective measurement on Bragg-scattered photons. Our scheme relies on coincident detection of two Bragg-scattered photons coming from two remote condensates in a Hanbury-Brown-Twiss-type experimental arrangement as schematically illustrated in Fig. 1. A and $B$ are two remote single-component condensates. Bragg scattering of pump photons occurs independently at the two 


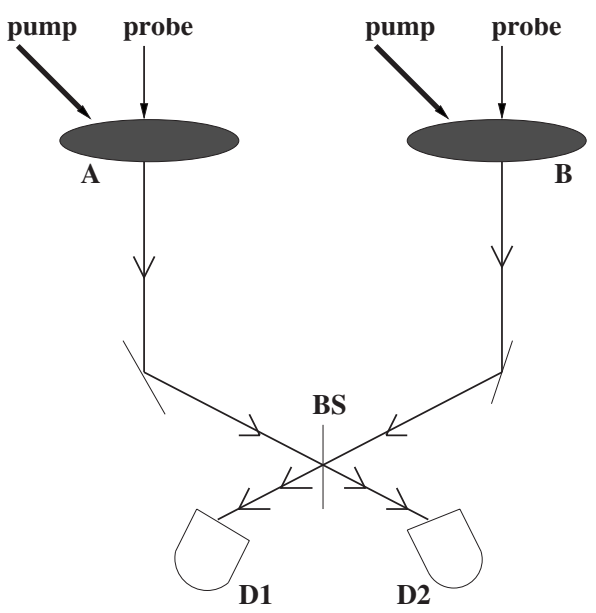

FIG. 1. A scheme for entangling two separate BECs by Bragg scattering and photodetection. $A$ and $B$ are two BECs which scatter photons from pump beams into probe ones. $D_{1}$ and $D_{2}$ are two photodetectors, and BS stands for beam splitter.

condensates, and the scattering is stimulated by probe beams. This gives rise to the generation of quasiparticles in both condensates in particular momentum modes determined by the relative angle between pump and probe light beams. The probe beams are assumed to have the same mode- that is, same frequency and polarization property. The scattered photons in the two probe modes are made to coalesce using a common beam splitter. Then the two photons coming out of the output ports of the beam splitter are detected by two photodetectors $D_{1}$ and $D_{2}$ in a coincident way. The joint projective state of the two spatially separate condensates is explicitly non-Gaussian. The entanglement of such nonGaussian states cannot be ascertained from the correlation in fluctuations of quadrature phase variables or number variables [39]. To prove the existence of entanglement in such a non-Gaussian state, we use the inequality criterion recently proposed by Agarwal and Biswas [39] based on the phase fluctuation of paring operators (of the form $\hat{a} \hat{b}$, where $\hat{a}$ and $\hat{b}$ are annihilation operators of the two subsystems) and the concept of partial transpose of Peres [40] and Horodecki et al. [41]. Shchukin and Vogel [42] and also Hillery and Zubairy [43] have recently introduced a class of similar inequalities, the violation of which is sufficient to show the presence of entanglement in two bosonic fields.

The paper is organized in the following way. Since Bragg scattering holds the key for generating quasiparticles and atom-photon entanglement, we first give a brief introduction to Bragg scattering in Sec. II in order to reveal the essential physical processes involved in Bragg scattering. We then formulate our theoretical model of projective measurement and discuss its effect in generating entanglement in Sec. III. In Sec. IV, we characterize entanglement and discuss our results. The paper is concluded in Sec. V.

\section{BRAGG SCATTERING}

In a Bose condensate of weakly interacting atomic gases, the zero-momentum $(\mathbf{k}=0)$ state is macroscopically occu- pied. Therefore, an atom-atom collision occurs primarily between zero- and nonzero-momentum atoms. In stimulated Raman or Bragg scattering, two far-off resonant laser beams with a small frequency difference are impinged on a trapped BEC. There are basically two physical processes in Bragg scattering. In the first process, a photon from the laser beam with higher frequency is scattered into a photon of the other laser mode. This causes a transformation of a zeromomentum atom into an atom of momentum $\mathbf{q}$, where $\mathbf{q}$ is the difference in photon momentum of the two beams. In the second process, an atom moving with a momentum $-\mathbf{q}$ is scattered into a zero-momentum state. Because of bosonic stimulation, the scattering of atoms from the zero- to q-momentum state will be the dominant process. There also occur processes which are opposite to the above two processes, but these are subdued due to phase mismatch. Thus Bragg scattering generates quasiparticles [34], predominantly in two momentum side modes $\mathbf{q}$ and $-\mathbf{q}$. Bragg spectroscopy [35] with coherent or classical light produces coherent states of quasiparticles in a BEC. When these quasiparticles are projected into the particle domain, they form two-mode squeezed and entangled states [25] in particle number variables.

Two remote condensates $A$ and $B$ are subjected to Bragg scattering with pairs of Bragg pulses. The frequencies and the directions of propagation of the laser beams are chosen such that Bragg resonance (phase matching) conditions of scattering in both the condensates are fulfilled. We assume that the pulse with higher frequency has a higher intensity and hence can act as a pump. The other laser beam which acts as a stimulant for scattering is of much lower intensity and so can be considered as a probe beam. We treat pump beams classically. Since scatterings at the two condensates occur independently, the Hamiltonian is simply the sum of the Hamiltonian $H^{A}$ and $H^{B}$ corresponding to condensates $A$ and $B$, respectively. Let $\hat{a}_{q}$ and $\hat{b}_{q}$ represent the annihilation operators for particles with momentum $\mathbf{q}$ in condensates $A$ and $B$, respectively. Let the corresponding Bogoliubov quasiparticles be denoted by $\hat{\alpha}_{q}$ and $\hat{\beta}_{q}$, respectively. In terms of these quasiparticle operators, the effective Hamiltonian as derived in the Appendix can be written as

$$
\begin{aligned}
H_{e f f}^{J}= & \hbar \omega_{q}^{B}\left(\hat{\chi}_{\mathbf{q}}^{\dagger} \hat{\chi}_{\mathbf{q}}+\hat{\chi}_{-\mathbf{q}}^{\dagger} \hat{\chi}_{-\mathbf{q}}\right)-\hbar \delta_{j} \hat{c}_{j}^{\dagger} \hat{c}_{j} \\
& +\left[\hbar \eta \hat{c}_{j}^{\dagger}\left(\hat{\chi}_{\mathbf{q}}^{\dagger}+\hat{\chi}_{-\mathbf{q}}\right)+\text { H.c. }\right],
\end{aligned}
$$

where the superscript $J$ stands for condensate $A$ or $B$ and $\hat{c}_{j}$ (with $j=a, b$ ) is the photon annihilation operator for the probe beam applied to condensate $J$. The particle operators $\hat{\pi}_{q}\left(\equiv \hat{a}_{q}, \hat{b}_{q}\right)$ are related to the quasiparticle operators $\hat{\chi}_{q}\left(\equiv \hat{\alpha}_{q}, \hat{\beta}_{q}\right)$ by the Bogoliubov's transformation

$$
\hat{\pi}_{q}=u_{q} \hat{\chi}_{q}-v_{q} \hat{\chi}_{-q}^{\dagger},
$$

with

$$
v_{q}^{2}=\left(u_{q}^{2}-1\right)=\frac{1}{2}\left(\frac{\hbar \omega_{q}+\mu}{\hbar \omega_{q}^{B}}-1\right)
$$

and 


$$
\hbar \omega_{q}^{B}=\left[\left(\hbar \omega_{q}+\mu\right)^{2}-\mu^{2}\right]^{1 / 2}
$$

is the energy of Bogoliubov's quasiparticle. Here $\hbar \omega_{q}$ $=\hbar^{2} q^{2} /(2 m)$ is the kinetic energy of a single atom, $\mu=\frac{\hbar^{2} \xi^{-2}}{2 m}$ is the chemical potential with $\xi=\left(8 \pi n_{0} a_{s}\right)^{-1 / 2}$ being the healing or coherence length, $\delta_{j}$ is the detuning between the pump and probe frequencies, and $\eta=\sqrt{N} f_{q} \Omega$, where $f_{q}=u_{q}-v_{q}$ and $\Omega$ is the two-photon Rabi frequency. We assume the Bragg resonance condition $\left(\delta \simeq \omega_{q}\right)$. The Hamiltonian can be solved exactly in the Heisenberg picture. The Heisenberg equations of motion for a triad of operators $X=\left(\hat{\alpha}_{\mathbf{q}} \hat{\alpha}_{-\mathbf{q}}^{\dagger} \hat{c}_{j}^{\dagger}\right)^{T}$ can be written in a matrix form $\dot{X}=i \omega_{q}^{B} \mathbf{M} X$, where $\mathbf{M}$ is a $3 \times 3$ matrix:

$$
\mathbf{M}=\left(\begin{array}{ccc}
-1 & 0 & -\tilde{\eta} \\
0 & 1 & \tilde{\eta} \\
\tilde{\eta}^{*} & \tilde{\eta}^{*} & -\tilde{\delta}
\end{array}\right),
$$

where $\tilde{x}=\tilde{x} / \omega_{q}^{B}$. Let $\mathbf{D}$ be the diagonalizing matrix of $\mathbf{M}$. The solutions can be explicitly written as

$$
X(t)=\mathbf{D E}(t) \mathbf{D}^{-1} X(0),
$$

where $\mathbf{E}$ is a diagonal matrix: $\mathbf{E}=\operatorname{diag}\left(\exp \left(i \lambda_{1} \tau\right)\right.$, $\left.\exp \left(i \lambda_{2} \tau\right), \exp \left(i \lambda_{3} \tau\right)\right)$, with $\tau=\omega_{q}^{B} t$ and $\lambda_{i}$ being the eigenvalues of the $\mathbf{M}$ matrix.

\section{ENTANGLEMENT PRODUCED BY PHOTODETECTION}

Our proposed scheme is shown in Fig. 1. Quasiparticles are generated in the condensates $A$ and $B$ due to stimulated light scattering in a pump-probe-type Bragg-spectroscopic method. Let $\hat{c}_{a}$ and $\hat{c}_{b}$ denote annihilation operators for the two probe light beams scattered by condensates $A$ and $B$, respectively. Using Eq. (6), the scattered light at the output of the two condensates can be represented by

$$
\begin{aligned}
& \hat{c}_{a}(t)=a_{q}(t) \hat{\alpha}_{q}^{\dagger}+a_{-q}(t) \hat{\alpha}_{-q}+a_{c}(t) \hat{c}_{a}(0), \\
& \hat{c}_{b}(t)=b_{q}(t) \hat{\beta}_{q}^{\dagger}+b_{-q}(t) \hat{\beta}_{-q}+b_{c}(t) \hat{c}_{b}(0),
\end{aligned}
$$

where $a_{ \pm q}, b_{ \pm q}, a_{c}$, and $b_{c}$ are time-dependent coefficients determined by Eq. (6). The scattered light output coming from the two condensates are passed through a beam splitter and finally collected at the two detectors at $D 1$ and $D 2$ as shown in Fig. 1. Let the reflectivity and transmissivity at left side of the beam splitter be $r$ and $t$, respectively, while those at right side $r^{\prime}$ and $t^{\prime}$. Then the photon annihilation operators at $D 1$ and $D 2$ can be expressed as

$$
\begin{aligned}
& \hat{C}_{D_{1}}=t^{\prime} \hat{c}_{b}+r \hat{c}_{a}, \\
& \hat{C}_{D_{2}}=t \hat{c}_{a}+r^{\prime} \hat{c}_{b} .
\end{aligned}
$$

Let the initial state of the total system-i.e., two condensates plus the two probe fields-be represented by

$$
\left|\Psi_{0}\right\rangle=|0,0\rangle_{A B}|\alpha, \beta\rangle_{\text {fields }},
$$

where $|0,0\rangle_{A B}$ indicates a product state with both condensates in the ground states of the quasiparticles, where first
" 0 " corresponds to condensate $A$ and the second " 0 " to condensate $B$. We assume that both probe fields are in coherent states $|\alpha, \beta\rangle_{\text {fields }}$ where the field amplitudes $\alpha$ and $\beta$ correspond to the probes incident at $A$ and $B$, respectively. Measurement of two-photon correlation via coincident detection of scattered probe lights at the two detectors will project the two-condensate density operator into

$$
\rho_{A B}=\mathcal{N} \operatorname{Tr}_{\text {fields }} \hat{C}_{D_{2}} \hat{C}_{D_{1}} \rho_{0} \hat{C}_{D_{1}}^{\dagger} \hat{C}_{D_{2}}^{\dagger},
$$

where $\operatorname{Tr}_{\text {fields }}$ implies tracing over the field states, $\rho_{0}$ $=\left|\Psi_{0}\right\rangle\left\langle\Psi_{0}\right|$, and $\mathcal{N}$ denotes a normalization factor. Now, substituting Eqs. (9) and (10) into Eq. (12) and using relations (7) and (8), we obtain $\rho_{A B}=|\Phi\rangle\langle\Phi|$, where

$$
\begin{aligned}
|\Phi\rangle= & \sqrt{\mathcal{N}}\left\langle\alpha, \beta\left|\hat{C}_{D_{2}} \hat{C}_{D_{1}}\right| \Psi_{0}\right\rangle \\
= & \sqrt{\mathcal{N}}\left[r^{\prime} t^{\prime}\left|0_{A}, S_{B}\left(1_{q}, 2_{q}\right)\right\rangle+r t\left|S_{A}\left(1_{q}, 2_{q}\right), 0_{B}\right\rangle\right] \\
& +\sqrt{\mathcal{N}}\left(r^{\prime} r+t^{\prime} t\right)\left|\Sigma_{A}\left(1_{q}\right), \Sigma_{B}\left(1_{q}\right)\right\rangle .
\end{aligned}
$$

The states $\left|S_{j}\left(1_{q}, 2_{q}\right)\right\rangle$ denote a superposition state of ground and one- and two- $q$-phonon excited states of condensate $j$ $(\equiv A, B)$. Similarly, $\left|\Sigma_{j}\left(0,1_{q}\right),\right\rangle$ is another superposition state of ground and one-phonon excited states of condensate $j$. Explicitly, these superposition states can be expressed as

$$
\begin{gathered}
\left|S_{A}\right\rangle=\sqrt{2} a_{q}^{2}\left|2_{q}\right\rangle_{A}+2 a_{q} a_{c} \alpha\left|1_{q}\right\rangle_{A}+a_{c}^{2} \alpha^{2}|0\rangle_{A}, \\
\left|\Sigma_{A}\right\rangle=a_{q}\left|1_{q}\right\rangle_{A}+a_{c} \alpha|0\rangle_{A} .
\end{gathered}
$$

Now, we have the reciprocity relations

$$
\begin{gathered}
r^{*} t^{\prime}+r^{\prime} t^{*}=0, \quad r^{*} t+r^{\prime} t^{*}=0, \\
\left|r^{\prime}\right|=|r|, \quad\left|t^{\prime}\right|=|t|, \quad|r|^{2}+|t|^{2}=1 .
\end{gathered}
$$

Let $t=|t| \exp (i \phi)$ and $t^{\prime}=\left|t^{\prime}\right| \exp \left(i \phi^{\prime}\right)$. Since phase changes by $\pi / 2$ on reflection, we have $r=i|r| \exp (i \phi)$ and $r^{\prime}$ $=i\left|r^{\prime}\right| \exp \left(i \phi^{\prime}\right)$. Using the reciprocity relations and considering the field amplitudes $\alpha$ and $\beta$ as real quantities, we obtain

$$
\begin{aligned}
|\Phi\rangle= & \sqrt{\mathcal{N}} r|| t\left|\left[\exp \left(2 i \phi^{\prime}\right)\left|0_{A}, S_{B}\right\rangle+\exp (2 i \phi)\left|S_{A}, 0_{B}\right\rangle\right]\right. \\
& +\sqrt{\mathcal{N}} \exp \left[i\left(\phi+\phi^{\prime}\right)\right]\left(|r|^{2}-|t|^{2}\right)\left|\Sigma_{A}, \Sigma_{B}\right\rangle .
\end{aligned}
$$

For a 50:50 beam splitter, we then have

$$
|\widetilde{\Phi}\rangle=\sqrt{\mathcal{N}} \frac{1}{2}\left[\left|0_{A}, S_{B}\right\rangle+\exp (2 i \Delta \phi)\left|S_{A}, 0_{B}\right\rangle\right]
$$

where $\Delta \phi=\phi-\phi^{\prime}$ and $|\widetilde{\Phi}\rangle=\exp \left(-2 i \phi^{\prime}\right)|\Phi\rangle$. Equation (17) is manifestly an entangled state of the two condensates $A$ and $B$. This state obtained via two-photon detection is different from the Gaussian state of each independent Bose condensate. Furthermore, the basis states involved in this entanglement describe collective modes or phonon modes of the condensates; $\left|0_{A}, S_{B}\right\rangle$ refers to a joint condensate state in which condensate $A$ is in a zero-phonon state and condensate $B$ is in a superposition of zero-, one-, and two-phonon states. This state results from quantum interference of two probable processes which are that (a) two scattered photons come from condensate $B$ and no photon is scattered by condensate $A$; 
these two photons are then split by the beam splitter and detected at the two detectors projecting the joint condensate state into the form $\left|0_{A}, S_{B}\right\rangle$. (b) The second process consists of scattering of two photons by condensate $A$ and no photon by condensate $B$; this results in the joint condensate state $\left|S_{A}, 0_{B}\right\rangle$. Since these two processes are probabilistic, the quantum interference of these two processes eventually gives rise to the resultant state of the form (17) for a 50:50 beam splitter. When one scattered photon comes from $A$ and another from $B$, there is the probability amplitude of $r r^{\prime}$ that both the detectors will detect the only reflected part of the two photons and also the probability amplitude of $t t^{\prime}$ that only transmitted part of both the photons will be detected. Both these processes project the joint condensate state into the form $\left|\Sigma_{A}\left(1_{q}\right), \Sigma_{B}\left(1_{q}\right)\right\rangle$. The net probability amplitude being the sum of these two quantities, for a 50:50 beam splitter they cancel each other. This explains why there is no component of $\left|\Sigma_{A}\left(1_{q}\right), \Sigma_{B}\left(1_{q}\right)\right\rangle$ in Eq. (17).

As discussed earlier, photons scattered by a condensate share entanglement with phonons or condensate momentum modes. These scattered photons can act as the carriers of quantum information of phonons. In a BEC of weakly interacting atomic gases, phonons are long lived. By coincident detection of two independent photons, but separately entangled with their respective scatterer condensates, we can establish a quantum communication channel between two condensates. Thus our proposed method of generating entanglement between two remote condensates may also find application in quantum cryptography [44] and teleportation [45]. Furthermore, this two-photon detection scheme may enable a partial Bell-state analysis [20,46,47]. Most of the earlier proposals for creating entanglement between two distant ions or atomic ensembles via photodetection are based on the electronic excitation and subsequent emission of photons that are detected. In such situations, spontaneous decay to unwanted electronic states and decoherence cannot be avoided. In our proposed scheme, since we use far-off resonant-stimulated Raman-type light scattering, spontaneous emission is negligible. Since the system is a Bose condensate of weakly interacting ultracold atoms with long coherence time, decoherence is also at a minimum level. Moreover, we use coherent light for stimulating photon scattering and so our scheme does not require any cavity.

\section{CHARACTERIZING ENTANGLEMENT IN STATE (17)}

\section{A. Negativity of the partial transpose of the density matrix}

The necessary and sufficient condition for entanglement in any bipartite system is the negativity of at least one of the eigenvalues of the partial transpose [40] of the density matrix of the system. Let $\rho^{A, B}$ denote the density matrix of a bipartite system composed of subsystems $A$ and $B$. Under partial transpose of Peres and Horodecki over the subsystem $B$ (or $A$ ), let the density matrix be represented by $\rho^{A, B^{T}}$ (or $\rho^{A^{T}, B}$ ) which can be derived by making transpose only on the operators of $B$ (or $A$ ). If the wave function of the composite system is inseparable, then there will be at least one eigenvalue of the transposed matrix which is negative.

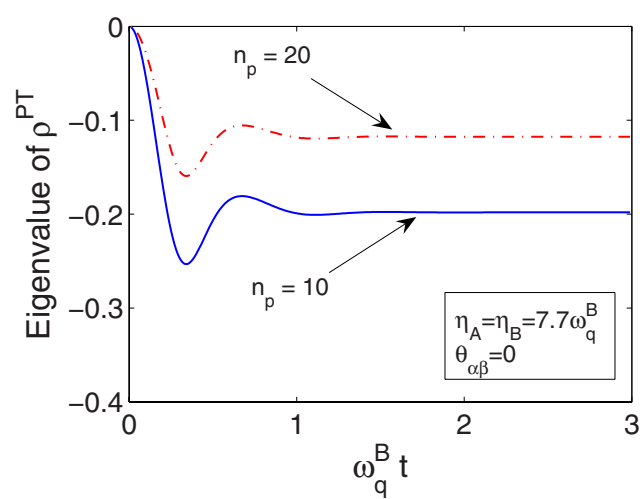

FIG. 2. (Color online) One eigenvalue of the partial-transposed density matrix $\rho^{A, B^{T}}$ calculated in the quasiparticle picture is plotted as a function of dimensionless interaction time $\omega_{q}^{B} t$, for different values of average probe photon numbers $n_{p}=10$ (solid line) and $n_{p}=20$ (dash-dotted line). The phase difference is $\Delta \phi=0$ and the phase difference between the two input laser fields $\theta_{\alpha \beta}=0$. The effective atom-field coupling constants are $\eta_{B}=\eta_{A}=7.7 \omega_{q}^{B}$.

Now, the state in Eq. (17) can be expressed as

$$
\left.\left.|\widetilde{\Phi}\rangle=C_{0}|0,0\rangle+\sum_{m=1}^{2}\left[\left|C_{m, 0}\right| m, 0\right\rangle+\left|C_{0 m}\right| 0, m\right\rangle\right],
$$

where $|m, n\rangle$ represents a joint quasiparticle number basis with $m$ number of quasiparticles in condensate $A$ and $n$ number of quasiparticles in condensate $B$. From Eqs. (14) and (17), we find

$$
C_{0}=a_{c}^{2} \alpha^{2}+\exp (2 i \Delta \phi) b_{c}^{2} \beta^{2} .
$$

Similarly, all other coefficients $C_{m, n}$ can be deduced from Eqs. (14) and (17). The density operator is $\rho^{A B}=|\tilde{\Phi}\rangle\langle\tilde{\Phi}|$. Taking the partial transpose on $\rho^{A B}$ with respect to $B$ implies changing the base operators $|i j\rangle\langle m n|\rightarrow| i n\rangle\langle m j|$ and the resulting matrix is $\rho^{A B^{T}}$. We numerically find that in all parameter regimes there is one eigenvalue of $\rho^{A B^{T}}$ which is always negative and hence the state (17) is an entangled state. Figure 2 shows that the negativity of the eigenvalue is more prominent when the probe photon number $|\alpha|^{2}=|\beta|^{2}=n_{p}$ is lower.

\section{B. Violation of the entanglement inequalities for observables}

Based on the idea of partial transpose, Simon and also Duan et al. [48] have independently given an entanglement criterion. Using variances in quadrature variables, an entanglement parameter [48] can be defined as

$$
\xi_{X P}=\frac{1}{2}\left[\left\langle\Delta\left(\hat{X}_{A}+\hat{X}_{B}\right)^{2}\right\rangle+\left\langle\Delta\left(\hat{P}_{A}-\hat{P}_{B}\right)^{2}\right\rangle\right],
$$

where $\hat{X}_{S \equiv A, B}=(1 / \sqrt{2})\left(\hat{S}+\hat{S}^{\dagger}\right)$ and $\hat{P}_{S}=(1 / \sqrt{2} i)\left(\hat{S}-\hat{S}^{\dagger}\right)$, with $\hat{S}$ being any bosonic operator of the subsystem $S$. According to the criterion of [48], the condition for the occurrence of entanglement is $\xi_{X P}<1$. This condition is necessary and sufficient for Gaussian quadrature variables only. For nonGaussian states, this is only sufficient. There are certain non- 
Gaussian bipartite states which are conspicuously inseparable, but do not fulfill the condition $\xi_{X P}<1$. In the present context, the state (17) is manifestly a non-Gaussian state. This prompts us to look for other criteria $[39,42,43]$ based on higher-order moments of observables.

Let us now test whether the higher-order entanglement criterion introduced in Ref. [39] can reveal the entanglement in state (17). To this end, let us first discuss what this criterion is. Using quasiparticle operators $\hat{\alpha}$ and $\hat{\beta}$ [same as $\hat{\alpha}_{q}$ and $\hat{\beta}_{q}$ defined in Eq. (2), the subscript $q$ being omitted for simplicity], we construct the operators

$$
\begin{gathered}
K_{x}=\frac{1}{2}\left(\hat{\alpha}^{\dagger} \hat{\beta}^{\dagger}+\hat{\alpha} \hat{\beta}\right), \\
K_{y}=\frac{1}{2 i}\left(\hat{\alpha}^{\dagger} \hat{\beta}^{\dagger}-\hat{\alpha} \hat{\beta}\right), \\
K_{z}=\frac{1}{2}\left(\hat{\alpha}^{\dagger} \hat{\alpha}+\hat{\beta}^{\dagger} \hat{\beta}+1\right),
\end{gathered}
$$

which satisfy $\mathrm{SU}(1,1)$ algebra. These $\mathrm{SU}(1,1)$ operators are previously employed for studying higher-order squeezing. The Heisenberg uncertainty relation of these operators implies the inequality

$$
\Delta\left(\hat{\alpha}^{\dagger} \hat{\beta}^{\dagger}+\hat{\alpha} \hat{\beta}\right) \Delta\left(\frac{\hat{\alpha}^{\dagger} \hat{\beta}^{\dagger}-\hat{\alpha} \hat{\beta}}{i}\right) \geqslant\left\langle\hat{\alpha}^{\dagger} \hat{\alpha}+\hat{\beta} \hat{\beta}^{\dagger}\right\rangle .
$$

Let us now make partial transpose $\hat{\beta} \leftrightarrow \hat{\beta}^{\dagger}$. Under this partial transpose, the above inequality becomes

$$
\Delta\left(\hat{\alpha}^{\dagger} \hat{\beta}+\hat{\alpha} \hat{\beta}^{\dagger}\right) \Delta\left(\frac{\hat{\alpha}^{\dagger} \hat{\beta}-\hat{\alpha} \hat{\beta}^{\dagger}}{i}\right) \geqslant\left\langle\hat{\alpha}^{\dagger} \hat{\alpha}+\hat{\beta} \hat{\beta}^{\dagger}\right\rangle .
$$

After some simple algebra, we have

$$
\begin{gathered}
\Delta\left(\hat{\alpha}^{\dagger} \hat{\beta}+\hat{\alpha} \hat{\beta}^{\dagger}\right)^{2}=N_{2}+N+M, \\
\Delta\left(\frac{\hat{\alpha}^{\dagger} \hat{\beta}-\hat{\alpha} \hat{\beta}^{\dagger}}{i}\right)^{2}=N_{2}+N-M-4\left|\left\langle\hat{\alpha}^{\dagger} \hat{\beta}\right\rangle\right|^{2},
\end{gathered}
$$

where $N_{2}=2\left\langle\hat{\alpha}^{\dagger} \hat{\alpha} \hat{\beta}^{\dagger} \hat{\beta}\right\rangle, N=\left\langle\hat{\alpha}^{\dagger} \hat{\alpha}+\hat{\beta} \hat{\beta}^{\dagger}\right\rangle$ and

$$
M=\left\langle\hat{\alpha}^{\dagger 2} \hat{\beta}^{2}\right\rangle+\left\langle\hat{\alpha}^{2} \hat{\beta}^{\dagger 2}\right\rangle-\left\langle\hat{\alpha}^{\dagger} \hat{\beta}+\hat{\alpha} \hat{\beta}^{\dagger}\right\rangle^{2} .
$$

Collecting all these terms, inequality (25) can be expressed in the form

$$
\left(N_{2}+N+M\right)\left(N_{2}+N-M-4\left|\left\langle\hat{\alpha}^{\dagger} \hat{\beta}\right\rangle\right|^{2}\right) \geqslant|N|^{2} .
$$

Violation of this inequality means the occurrence of entanglement. It is worth mentioning here that all these criteria are sufficient for showing entanglement in a bipartite system.

We now concentrate on the form of the state given in Eq. (18). In all joint base states $|m, n\rangle$, the quasiparticle vacuum state $\left(|0\rangle_{S}\right)$ of either condensate $S(A, B)$ appears explicitly. This means that the correlation function $N_{2}$ of the quasiparticle number operators is zero for any parameter regime. This reduces inequality (29) to the form

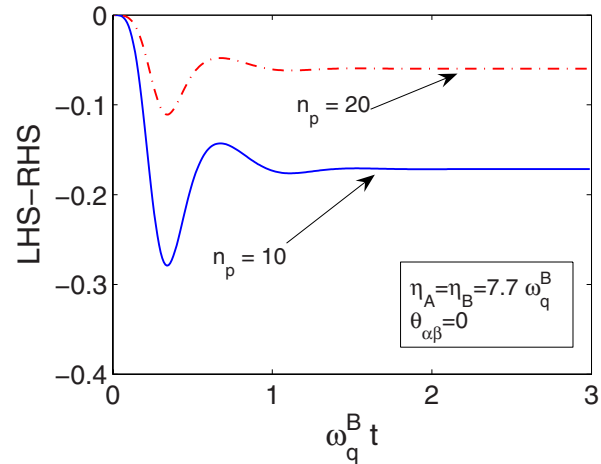

FIG. 3. (Color online) Left-hand side (LHS) minus right-hand side (RHS) of the entanglement inequality (29) calculated in the quasiparticle picture is plotted as a function of $\omega_{q}^{B} t$ for average probe photon numbers $n_{p}=10$ (solid line) and $n_{p}=20$ (dashed line). All other parameters are same as in Fig. 2.

$$
\left.-M^{2}-4(N+M)\left|\left\langle\hat{\alpha}^{\dagger} \hat{\beta}\right\rangle\right|^{2}\right) \geqslant 0 .
$$

Since $N_{2}=0$, the variance $\Delta\left(\hat{\alpha}^{\dagger} \hat{\beta}+\hat{\alpha} \hat{\beta}^{\dagger}\right)^{2}$ equals $(N+M)$. Because this variance is non-negative, the quantity $(N+M)$ must be non-negative. This implies that inequality (30) is violated. Thus we have proved that state (17) is entangled.

\section{Stronger entanglement by selection of probe phase}

Here we show that the entanglement can be made stronger by choosing appropriate phase of the two probe beams. In state (18), there is a purely Gaussian component corresponding to the basis $|0,0\rangle$ which implies that both condensates are in their respective quasiparticle vacuum. Since the criterion (29) is devised for verifying entanglement in a non-Gaussian bipartite state, we expect that, on elimination of this purely Gaussian component from (18), violation of the inequality (29) should be much stronger. This component can be eliminated if the coefficient $C_{0}$ given in Eq. (19) is made to vanish. For simplicity, let us assume that both condensates are identical and their atom-field coupling constants are the same. This means $a_{c}=b_{c}$. Furthermore, we assume that $\Delta \phi$ $=0$. In such a situation, to make $C_{0}$ vanish implies $\alpha^{2}=-\beta^{2}$ where $\alpha$ and $\beta$ are the amplitude of the two input probe fields which are assumed to be in the coherent states. In other words, this means $\alpha=i \beta$; that is, the two input probes should have a phase difference $\theta_{\alpha \beta}$ equal to $\pi / 2$. Now, a phase difference of $\pi / 2$ between two beams can easily be made if they are derived from a single laser through a 50:50 beam splitter with transmitted and reflected beams being used as the two input beams. We plot left-hand side (LHS) minus right-hand side (RHS) of Eq. (29) as a function of interaction time in Figs. 3 and 4 for various parameters. Obviously, negativity of LHS-RHS implies violation of inequality (30) and hence entanglement. For all our numerical illustrations, we consider two identically prepared BECs with the same atom-field coupling strength. However, it is worth pointing out that this assumption is not necessary for exploration of entanglement between the condensates; this is assumed only for the sake of simplicity. Figure 3 shows the effect of probe photon number (or intensity) on the degree of violation of 


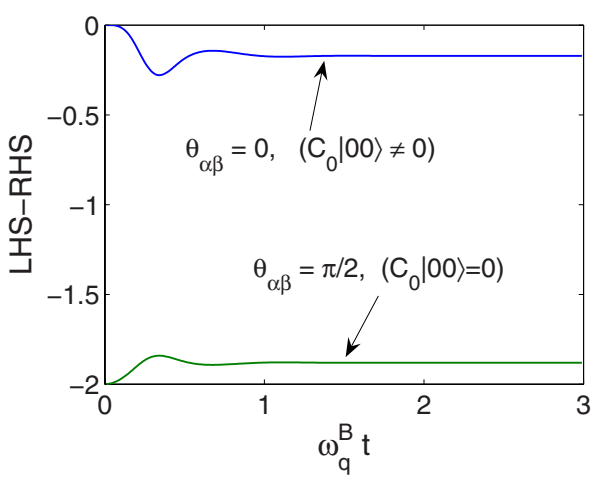

FIG. 4. (Color online) The effect of Gaussian component of the entangled state (17) on the violation of entanglement inequality (29) in the quasiparticle picture is illustrated. The upper curve corresponds to the case when the purely Gaussian basis state $|00\rangle$ is present in (17), and the lower curve corresponds to the case when this state is eliminated by choosing the appropriate phases of the laser fields. For two identical BECs and same atom-field couplings, when the phase difference between the two input probe beams is $\pi / 2$, the coefficient of the basis $|00\rangle$ vanishes provided the path difference between the two output probe beams arriving at the detectors is zero. Here $n_{p}=10$ and all other parameters are same as in Fig. 2.

the inequality. The weaker the probe intensity is, the stronger is the violation. Figure 4 illustrates that when the purely Gaussian component is eliminated $\left(\theta_{\alpha \beta}=\pi / 2\right)$ from the state (17), the negativity of LHS-RHS becomes much stronger, implying that the criterion (29) works better for highly nonGaussian entangled states. For identical coupling constants and detuning between the pump and the probe for the two condensates, we find that the degree of violation of the inequality saturates at the long interaction time limit. However, for any mismatch in coupling constants or detunings, we have found that LHS-RHS tends to zero in the long interaction time regime (not shown). The effect of atom-field coupling strength on the negativity of LHS-RHS is illustrated in Fig. 5. We notice that in the strong-coupling regime the negativity is stronger. Furthermore, in the limit of very strong coupling the violation of the inequality becomes al-

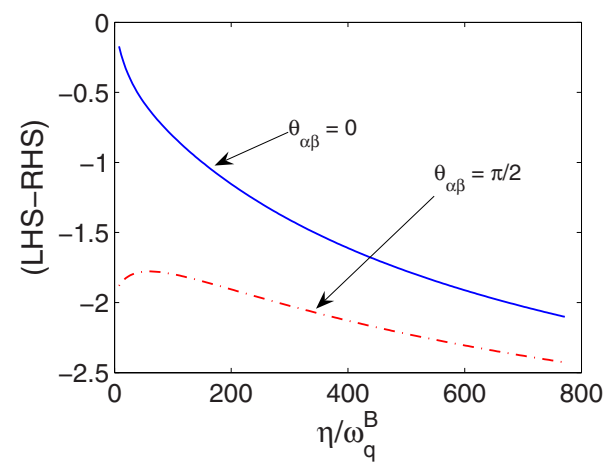

FIG. 5. (Color online) Violation of inequality (29) as a function of coupling constant $\eta_{A}=\eta_{B}=\eta$ for two cases $\theta_{\alpha \beta}=0$ (solid line) and $\theta_{\alpha \beta}=0$ (dashed line) which correspond to presence and absence of the purely Gaussian basis $|00\rangle$ in the entangled state. Here $\omega_{q}^{B} t$ $=5$ and $n_{p}=10$. most insensitive to the initial phase difference $\theta_{\alpha \beta}$ of the two probe beams. This indicates that strong coupling is important for entanglement between remote condensates. This in turn brings in the important role of condensates in quantuminformation science: Since the effective atom-field coupling strength is proportional to the square root of number of atoms, the strong-coupling regime can easily be attained with a $\mathrm{BEC}$ rather than a thermal gas. Since thermal and phase fluctuations in a BEC are at the minimum level, collective atomfield coupling can easily be accomplished with a BEC in a cavity with a moderate $Q$ factor. For experimental verification of entanglement, one needs to measure the various variances (appearing in the inequality) in the phonon or collective excitation modes of BEC.

\section{CONCLUSION}

In conclusion, we have shown that two remote independent condensates can be made entangled in collective excitations of BECs such as quasiparticle or phonon variables by projective measurement on two photons Bragg scattered by the two condensates. The generated entangled state is explicitly non-Gaussian and the existing criterion for entanglement in Gaussian variables is insufficient in revealing entanglement in this state. This has prompted us to test another criterion [39] introduced specially for testing entanglement in non-Gaussian states. We have shown that with this criterion the projective state of the two condensates is entangled and the entanglement can be made stronger by choosing the probe phase in such a way so that the non-Gaussian nature of the entangled state becomes more prominent. The entanglement is shown to be stronger when the probe beams are weaker and the atom-field coupling is higher. Since atoms behave collectively in BEC-field interactions and also because of $\sqrt{N}$ ( $N$ is the atom number) scaling of the coupling strength, BECs can play an important role in the generation and manipulation of entanglement. Since the entanglement in the present context arises in the higher-order fluctuations of the pairing operators, experimental detection of this entanglement requires new techniques that will enable one to measure the pairing fluctuations of phonons or quasiparticles. Using Bragg spectroscopy phonons in a BEC have been detected by Ketterle's group. But how to detect phononphonon correlation which is crucially required for exploration of higher-order entanglement is presently unknown. Perhaps by knocking out pairs of atoms from the two trapped condensates and measuring their fluctuations, one can detect this entanglement. Our proposed scheme can be easily generalized for multiple condensates. Furthermore, it may be interesting to generate entanglement in both spin and motional degrees of freedom using spinor condensates and polarized light in our scheme with the possibility of an interesting interplay of entanglement in spin and center-of-mass degrees of freedom.

\section{ACKNOWLEDGMENTS}

This work is supported by NSF Grant No. 0653494. One of us (B.D.) gratefully acknowledges the hospitality at Okla- 
homa State University where this work was initiated during his visit.

\section{APPENDIX}

Here we derive the effective Hamiltonian for Bragg scattering in a condensate. The total Hamiltonian of a condensate interacting with two single-mode light fields is $H=H_{A}+H_{F}$ $+H_{A F}$, where $H_{F}=\hbar \omega_{1} \hat{c}_{\mathbf{k}_{1}}^{\dagger} \hat{c}_{\mathbf{k}_{1}}+\hbar \omega_{2} \hat{c}_{\mathbf{k}_{2}}^{\dagger} \hat{c}_{\mathbf{k}_{2}}$ corresponds to the two fields described by the operators $\hat{c}_{\mathbf{k}_{1}}$ and $\hat{c}_{\mathbf{k}_{2}}$ with photon momenta $\mathbf{k}_{1}$ and $\mathbf{k}_{2}$ and frequencies $\omega_{1}$ and $\omega_{2}$, respectively. We assume $\omega_{1}>\omega_{2}$. The free part of the atomic Hamiltonian

$$
\begin{aligned}
H_{A}= & \sum_{k} \hbar \omega_{k} \hat{\pi}_{\mathbf{k}}^{\dagger} \hat{\pi}_{\mathbf{k}}+\frac{4 \pi \hbar^{2} a_{s}}{2 m V} \\
& \times \sum_{\mathbf{k}_{3}, \mathbf{k}_{4}, \mathbf{k}_{5}, \mathbf{k}_{6}} \hat{\pi}_{\mathbf{k}_{3}}^{\dagger} \hat{\pi}_{\mathbf{k}_{4}}^{\dagger} \hat{\pi}_{\mathbf{k}_{5}} \hat{\pi}_{\mathbf{k}_{6}} \delta_{\mathbf{k}_{3}+\mathbf{k}_{4}, \mathbf{k}_{5}+\mathbf{k}_{6}}
\end{aligned}
$$

governs the dynamics of a weakly interacting atomic condensate and

$$
H_{A F}=\hbar \Omega \hat{c}_{\mathbf{k}_{2}}^{\dagger} \hat{c}_{\mathbf{k}_{1}} \sum_{k}\left(\hat{\pi}_{\mathbf{q}+\mathbf{k}}^{\dagger} \hat{\pi}_{\mathbf{k}}+\hat{\pi}_{-\mathbf{q}+\mathbf{k}} \hat{\pi}_{\mathbf{k}}^{\dagger}\right)+\text { H.c. }
$$

describes the atom-field interaction. Here $\hat{\pi}_{k}\left(\hat{\pi}_{k}^{\dagger}\right)$ is the annihilation (creation) operator of an atom with momentum $\mathbf{k}$ and frequency $\omega_{k}=\frac{\hbar k^{2}}{2 m} ; \quad \mathbf{q}=\mathbf{k}_{1}-\mathbf{k}_{2}, \quad \Omega$ $=\left(\vec{E}_{1} \cdot \vec{d}_{13}\right)\left(\vec{E}_{2} \cdot \vec{d}_{32}\right) /\left(\hbar^{2} \Delta\right)$ is the two-photon Rabi frequency, where $E_{1(2)}$ are the field amplitudes, the $\vec{d}_{i j}$ is the electronic transition dipole moment between the states $|i\rangle$ and $|j\rangle$ of an atom, and $\Delta$ is the detuning of the first laser field (with frequency $\omega_{1}$ ) from the transition frequency between the electronic ground $(|1\rangle)$ and excited $(|3\rangle)$ levels of the atom. For a single-component condensate the electronic ground states $|1\rangle$ and $|2\rangle$ are the same. Here $a_{s}$ is the $s$-wave scattering length of the atoms and $V$ is the volume of the condensate.

Using Bogoliubov's prescription $\hat{\pi}_{0}, \hat{\pi}_{0}^{\dagger} \rightarrow \sqrt{N_{0}}$ and keeping the number density $n_{0}=N_{0} / V$ fixed in the thermodynamic limit, one can transform the Hamiltonian $H_{A}$ into a quadratic form. Further, applying Bogoliubov's transformation it is possible to diagonalize $H_{A}$ and rewrite the entire Hamiltonian in terms of Bogoliubov's quasiparticle operators $\hat{\chi}_{\mathbf{k}}$. Considering the condensate ground-state energy as the zero of the energy scale and treating the laser light with higher frequency $\left(\omega_{1}\right)$ classically, the effective Hamiltonian can be written as

$$
\begin{aligned}
H_{e f f}= & \hbar \omega_{q}^{B}\left(\hat{\chi}_{\mathbf{q}}^{\dagger} \hat{\chi}_{\mathbf{q}}+\hat{\chi}_{-\mathbf{q}}^{\dagger} \hat{\chi}_{-\mathbf{q}}\right)-\hbar \delta \hat{c}_{\mathbf{k}_{2}}^{\dagger} \hat{c}_{\mathbf{k}_{2}} \\
& +\left[\hbar \eta \hat{c}_{\mathbf{k}_{2}}^{\dagger}\left(\hat{\chi}_{\mathbf{q}}^{\dagger}+\hat{\chi}_{-\mathbf{q}}\right)+\text { H.c. }\right],
\end{aligned}
$$

where $\delta=\omega_{1}-\omega_{2}$ and $\eta=\sqrt{N} f_{q} \Omega$; where $f_{q}=u_{q}-v_{q}$. In writing the above equation, we have retained only two dominant momentum side modes of the condensate under the assumption of Bragg resonance $\left(\delta \simeq \omega_{q}\right)$.
[1] B. Julsgaard, A. Kozhekin, and E. S. Polzik, Nature (London) 413, 400 (2001).

[2] D. N. Matsukevich, T. Chaneliere, S. D. Jenkins, S. Y. Lan, T. A. B. Kennedy, and A. Kuzmich, Phys. Rev. Lett. 96, 030405 (2006).

[3] C. W. Chou et al., Nature (London) 438, 828 (2005).

[4] D. Bouwmeester, J. W. Pan, M. Daniell, H. Weinfurter, and A. Zeilinger, Phys. Rev. Lett. 82, 1345 (1999); J.-W. Pan, M. Daniell, S. Gasparoni, G. Weihs, and A. Zeilinger, ibid. 86, 4435 (2001); Z. Zhao et al., Nature (London) 430, 54 (2004); C.-Y. Lu et al., Nat. Phys. 3, 91 (2007).

[5] N. Kiesel, C. Schmid, G. Toth, E. Solano, and H. Weinfurter, Phys. Rev. Lett. 98, 063604 (2007).

[6] D. L. Moehring, P. Maunz, S. Olmschenk, K. C. Younge, D. N. Matsukevich, L.-M. Duan, and C. Monroe, Nature (London) 449, 68 (2007).

[7] J. Beugnon et al., Nature (London) 440, 779 (2006).

[8] P. Maunz et al., e-print arXiv:quant-ph/0608047.

[9] P. Maunz et al., Nat. Phys. 3, 1 (2007).

[10] C. Thiel, J. von Zanthier, T. Bastin, E. Solano, and G. S. Agarwal, Phys. Rev. Lett. 99, 193602 (2007).

[11] R. H. Dicke, Phys. Rev. 93, 99 (1954).

[12] J. K. Stockton, J. M. Geremia, A. C. Doherty, and H. Mabachi, Phys. Rev. A 67, 022112 (2003).

[13] M. Bourennane, M. Eibl, S. Gaertner, N Kiesel, C. Kurtsiefer, and H. Weinfurter, Phys. Rev. Lett. 96, 100502 (2006).

[14] G. Tóth, J. Opt. Soc. Am. B 24, 275 (2007).
[15] A. R. Usha Devi, R. Prabhu, and A. K. Rajagopal, Phys. Rev. Lett. 98, 060501 (2007); Phys. Rev. A 76, 012322 (2007).

[16] A. Retzker, E. Solano and B. Reznik, Phys. Rev. A 75, 022312 (2007).

[17] C. Cabrillo, J. I. Cirac, P. Garcia-Fernandez, and P. Zoller, Phys. Rev. A 59, 1025 (1999)

[18] S. Bose, P. L. Knight, M. B. Plenio, and V. Vednal, Phys. Rev. Lett. 83, 5158 (1999).

[19] C. Skornia, J. von Zanthier, G. S. Agarwal, E. Werner, and H. Walther, Phys. Rev. A 64, 063801 (2001).

[20] C. Simon and W. T. M. Irvine, Phys. Rev. Lett. 91, 110405 (2003).

[21] L.-M. Duan and H. J. Kimble, Phys. Rev. Lett. 90, 253601 (2003).

[22] L.-M. Duan et al., Nature (London) 414, 413 (2001).

[23] S. L. Braunstein and H. J. Kimble, Phys. Rev. Lett. 80, 869 (1998); G. J. Milburn and S. L. Braunstein, Phys. Rev. A 60, 937 (1999); T. Opatrny and G. Kurizki, Phys. Rev. Lett. 86, 3180 (2001); A. Kitagawa and K. Yamamoto, e-print arXiv:quant-ph/0202154.

[24] N. N. Bogoliubov, J. Phys. (Moscow) 11, 23 (1947).

[25] B. Deb and G. S. Agarwal, Phys. Rev. A 65, 063618 (2002).

[26] A. Einstein, B. Podolosky, and N. Rosen, Phys. Rev. 47, 777 (1935).

[27] A. Sorensen et al., Nature (London) 409, 63 (2001).

[28] L.-M. Duan, A. Sorensen, J. I. Cirac, and P. Zoller, Phys. Rev. Lett. 85, 3991 (2000). 
[29] H. Pu and P. Meystre, Phys. Rev. Lett. 85, 3987 (2000).

[30] K. Helmerson and L. You, Phys. Rev. Lett. 87, 170402 (2001).

[31] M. G. Moore and P. Meystre, Phys. Rev. A 59, R1754 (1999).

[32] S. Raghavan et al., Opt. Commun. 188, 149 (2001).

[33] S. Choi and N. P. Bigelow, Phys. Rev. A 72, 033612 (2005).

[34] D. M. Stamper-Kurn, A. P. Chikkatur, A. Gorlitz, S. Inouye, S. Gupta, D. E. Pritchard, and W. Ketterle, Phys. Rev. Lett. 83, 2876 (1999).

[35] J. Stenger, S. Inouye, A. P. Chikkatur, D. M. Stamper-Kurn, D. E. Pritchard, and W. Ketterle, Phys. Rev. Lett. 82, 4569 (1999); S. Inouye, R. F. Low, S. Gupta, T. Pfan, A. Gorlitz, T. L. Gustavson, D. E. Pritchard, and W. Ketterle, ibid. 85, 4225 (2000).

[36] T. Gasenzer, D. C. Roberts, and K. Burnett, Phys. Rev. A 65 , 021605(R) (2002); D. C. Roberts, T. Gasenzer, and K. Burnett, J. Phys. B 35, L113 (2002).

[37] B. Deb and G. S. Agarwal, Phys. Rev. A 67, 023603 (2003).

[38] M. Saba, T. A. Pasquini, C. Sanner, Y. Shin, W. Ketterle, and D. E. Pritchard, Science 307, 1945 (2005).
[39] G. S. Agarwal and A. Biswas, New J. Phys. 7, 211 (2005).

[40] A. Peres, Phys. Rev. Lett. 77, 1413 (1996).

[41] M. Horodecki, P. Horodecki, and R. Horodecki, Phys. Lett. A 223, 1 (1996).

[42] E. Shchukin and W. Vogel, Phys. Rev. Lett. 95, 230502 (2005).

[43] M. Hillery and M. S. Zubairy, Phys. Rev. Lett. 96, 050503 (2006).

[44] C. H. Bennett, G. Brassard, and N. D. Mermin, Phys. Rev. Lett. 68, 557 (1992); A. K. Ekert, ibid. 67, 661 (1991).

[45] C. H. Bennett, G. Brassard, C. Crepeau, R. Jozsa, A. Peres, and W. K. Wootters, Phys. Rev. Lett. 70, 1895 (1993).

[46] J. S. Bell, Physics (Long Island City, N.Y.) 1, 195 (1965).

[47] S. L. Braunstein and A. Mann, Phys. Rev. A 51, R1727 (1995); K. Mattle, H. Weinfurter, P. G. Kwiat, and A. Zeilinger, Phys. Rev. Lett. 76, 4656 (1996).

[48] L. M. Duan, G. Giedke, J. I. Cirac, and P. Zoller, Phys. Rev. Lett. 84, 2722 (2000); R. Simon, ibid. 84, 2726 (2000). 